\title{
28 Resarch Square \\ The possibility of a novel warm-up strategy using capacitive and resistive electric transfer
}

\section{michio wachi ( $\square$ m-wachi@pt-si.aino.ac.jp )}

Biwako Professional University of Rehabilitation: Biwako Rehabilitation Senmonshoku Daigaku

\section{Takumi Jiroumaru}

Biwako Professional University of Rehabilitation

\section{Ayako Satonaka}

Biwako Professional University of Rehabilitation: Biwako Rehabilitation Senmonshoku Daigaku

\section{Masae lkeya}

Biwako Professional University of Rehabilitation: Biwako Rehabilitation Senmonshoku Daigaku

\section{Oka Yasumasa}

kanazawa orthopaedic \& sports medicine clinic

\section{Takamitsu Fujikawa}

Bukkyo University: Bukkyo Daigaku

\section{Research Article}

Keywords: capacitive and resistive electric transfer, muscular stiffness, range of motion, active straight leg raise, multifidus muscle

Posted Date: February 18th, 2022

DOI: https://doi.org/10.21203/rs.3.rs-1260390/v1

License: (1) (i) This work is licensed under a Creative Commons Attribution 4.0 International License.

Read Full License 


\section{Abstract}

Background: Capacitive and resistive electric transfer (CRET) is becoming popular in sports settings. Although CRET is effective for improving pain and healing injured tissues, its influence on muscle function and morphology is still unclear. This study confirmed the immediate effects of CRET on the duration of muscular stiffness and the range of motion (ROM).

Methods: This paper describes the protocol for a single arm trial with non-blinding of participants and researcher. Twenty-four healthy men participated in the study. They received CRET therapy for their lower back areas. The muscular stiffness of the multifidus muscle (superficial and deep) and the ROM using the active straight leg raise (ASLR) test were measured pre-intervention, post-intervention (immediately), and 15 minutes and 30 minutes post-intervention. We compared these parameters with one-way analysis of variance and Dunnett's test (multiple comparison tests for subtests).

Results: The muscular stiffness of the superficial and deep multifidus muscles became significantly lesser, and the ASLR test showed a significant increase compared to that obtained at pre-intervention. In addition, these effects persisted for 30 minutes.

Conclusions: Warm-up is vital for improving muscular stiffness and for increasing the ROM. CRET is a useful device to achieve these aims as a passive warm-up method in sports settings.

Trial registration number: UMIN000046304. Registered 7 December 2021 - Retrospectively registered, https://upload.umin.ac.jp/cgi-open-bin/ctr_e/ctr_view.cgi?recptno=R000052845

\section{Background}

Thermal stimulation to the human body results in vasodilation and improves blood circulation [1-4]. Thermotherapy is used as one of the physiotherapy methods in clinical settings; its effectiveness in the treatment of musculoskeletal pain and tissue injury (muscles, tendons, and ligaments) is well known [14]. Therefore, thermotherapy is recognized as a major modality for therapy. Thermotherapy is classified as superficial or deep thermotherapy. Generally, superficial thermotherapy includes heating pads, hot packs, paraffin bath, and infrared heat lamps and are useful for increasing the skin and surface tissue temperature along with vasodilation. However, deep thermotherapy techniques, such as shortwave diathermy, microwave diathermy, and ultrasound, also produce vasodilation but these can increase the deep tissue temperature [5]. It is reported that deep thermotherapy devices, such as ultrasound and shortwave diathermy, can improve hemoglobin saturation, boost the treatment, and improve fatigue [6, 7].

Blood (hemoglobin) delivers oxygen to all the parts of the body and carries carbon dioxide back to the lungs. The oxygenated hemoglobin and muscle blood volume are reportedly decreased in fatigued lumbar muscles [8]. There have been reports that recovered tissue oxygenation facilitates the improvement of fatigued muscles [9-11]. Vasodilation due to heat is produced through the direct activation of the skin thermoreceptors in vascular smooth muscles and the suppression of the 
sympathetic nervous system via the indirect activation of local spinal reflexes and increased levels of inflammatory chemical mediators [12-14]. These factors combined cause vasodilation.

Traditional deep thermotherapy techniques have several disadvantages. For instance, ultrasound has a risk of periostitis due to the energy concentration on the bone tissues [15]. Diathermy devices with a frequency of 8-14 MHz may cause excessive heat during the treatment and result in skin burns [16]. Recently, capacitive and resistive electric transfer (CRET) with a frequency of 400-500 kHz has been developed [17]. It uses high-frequency electromagnetic energy to generate heat within the body tissues. CRET with a lower frequency does not produce excessive heat between the skin and electrode; therefore, it is safer than traditional diathermy. Furthermore, previous studies have shown that CRET is effective in improving low back or shoulder pain by increasing the hemoglobin saturation and muscle temperature [18-20]. Although CRET is effective for improving pain and healing injured tissues, its influence on muscle function and morphology is still unclear.

Increased muscle temperature affects the maximum muscle power and maximum contraction speed. McGowan et al. reviewed that the raised muscle temperature (passive or active) leads to an increase in adenosine triphosphate turnover, cross-bridge cycling rate, and muscle fiber conduction velocity [21]. Athletes need splinting after sustaining high-intensity injuries and could benefit using this mechanism; hence, therapeutic modalities, including CRET, are frequently used. In the Rio 2016 Olympic games, 28\% of athletes received electrotherapy to recover from injuries and maintain their body at the Olympic Polyclinic [22]. This percentage was the most frequent among all the treatment modalities. Compared to the London 2012 Olympic games, the type and utilization of modality treatment had increased in the Rio 2016 Olympic games [23]; hence, these therapies are more popular in sports settings. If the CRET enables an immediate rise in the temperature of the deep tissues and contributes to improved physical function, then this device is useful for warm-up before sports. In this study, we examined the immediate effects, such as the ROM and the duration of the multifidus muscle stiffness, after irradiating the low back area using CRET.

\section{Materials And Methods}

\section{Participants}

Participants were recruited from four hospitals and a university. The inclusion criteria were as follows: age of 20-50 years and male. The rehabilitation staff confirmed the participant's health condition using a questionnaire, and the participant attended as a volunteer. The exclusion criteria were as follows:

presence of tumors, obvious lumbar herniation, lumbar spinal canal stenosis, infections, musculoskeletal disorder, sciatic nerve compression and pain, sensory impairment, and decreased muscle strength and reflexes. In addition, participants did not have a habit of performing strenuous exercise. Each participant signed an informed consent form, based on the requirements of the Helsinki Declaration. This study was approved by the ethics committee of the Kanazawa Orthopedic Sports Medicine Clinic (kanazawa-OSMC- 
2021-004) and registered in the University Hospital Medical Information Clinical Trials Registry (UMIN000046304).

This interventional study is a single arm trial with non-blinding of participants and researcher. We calculated the sample size needed for one-way analysis of variance (ANOVA) (effect size 0.80, significance level $(a)$ error $=0.05$, power $(1-\beta)=0.80$ ) using $G^{*}$ Power software (version 3.1 ; Heinrich Heine University of Düsseldorf, Düsseldorf, Germany), and obtained a result of 24.

\section{Experimental procedure}

Participants' body mass index (BMI) was calculated after measuring their height and weight. They also received CRET therapy once on their lower back. The stiffness of the superficial and deep multifidus muscles on the right side was measured. Subsequently, the range of motion (ROM) using active straight leg raise (ASLR) was measured. These measurements were conducted at baseline (T0), immediately after intervention (T1), 15 minutes after intervention (T2), and 30 minutes after intervention (T3).

\section{Intervention}

The CRET device was Physio Radio Stim Pro (SAKAI Medical Co. Ltd., Tokyo, Japan). This device works at a frequency of $500 \mathrm{kHz}$. A highly rigid circular electrode was used as the active electrode, and a large, flexible rectangular electrode was used as the inactive electrode. This device supplies high-frequency electromagnetic energy with two modes for the active electrode: capacitive electrode transfer (CET) and resistive electrode transfer (RET). The CET electrode is coated with polyamide, which can insulate from the skin surface as a dielectric medium and heat on the external skin. The uncoated RET electrode supplies heat to the deep tissues of the body owing to the direct conduction of energy to the inactive electrode.

Participants received CRET therapy in the prone position on the plinth. An inactive electrode was placed on the abdominal area. To maintain conductivity between the electrode and skin surface during the intervention, a manufacturer-supplied cream was used. The irradiation time of CRET was 5 minutes by CET and 10 minutes by RET for the multifidus and erector spinae muscles. This intervention time was based on previous studies $[7,20]$. The intensity of CRET was defined as six or seven using the 11-level analog scale $(0=$ no heat sensing, $10=$ worst heat sensing $)$ to demonstrate the participants' subjective feeling of heat. After 15 minutes of intervention, the conductive cream was completely removed. No other therapies that might affect the judgment of the therapeutic efficacy were conducted.

\section{Measurements}

\section{Ultrasound}

We used a B-mode ultrasonographic apparatus (SSD-3500SV; FUJIFILM Healthcare Corporation, Japan) with a linear transducer (scanning frequency, 7.5 MHz). An attachment dedicated to an acoustic coupler (EZU-TECPL1, FUJIFILM Healthcare Corporation, Tokyo, Japan) with an elastic modulus of $22.6 \pm 2.2 \mathrm{kPa}$ was set on the probe and placed between the measurement area and probe. The elastography images 
were recorded while pressing the transducer attached to the acoustic coupler on the multifidus muscle. Each participant in the prone position placed a rolled towel under the abdomen to adjust the neutral alignment of the lumbar spine. The stiffness of the superficial and deep multifidus muscles was measured at $2 \mathrm{~cm}$ on the right side of the third lumbar spine vertebra, at the space between the third and the fourth lumbar spine vertebra transverse processes. Owing to the anisotropy of muscles, the parallel direction of the muscle fiber was confirmed [24]. Subsequently, the outline of the probe was marked on the skin for the accuracy of each measurement. All measurements were performed by an experienced rater.

In previous studies, muscular stiffness was measured as the strain ratio, calculated by using an acoustic coupler, that acts as the reference $[25,26]$. The measurement area of the acoustic coupler was defined as follows: the vertical axis is the vertical diameter of the acoustic coupler, and the horizontal axis is the central 3/4 area of the image. For the measurement area of the multifidus muscle, the vertical axis is the muscle thickness, and the horizontal axis is the central 3/4 area of the image. Regarding muscular stiffness, the measurement area of each muscle $(A)$ was divided by the measurement area of the acoustic coupler (B) and calculated (A/B) for the value. If the strain ratio is larger than 1.0, the muscle is stiffer than the coupler $(>22.6 \mathrm{kPa})$ (Figure 1).

\section{ASLR test}

The participants lay down in a supine position on the plinth. Both arms were rested on the abdomen, and the cervical spine was maintained in a neutral position using a towel under the head. The examiner asked the participant to raise their right leg slowly with the hip joint in a neutral position, knee joint in extension, and ankle in maximum dorsiflexion $[27,28]$. The participant was asked to stop raising the leg at 'submaximal pain,' and at this point, ROM was measured. For measurement of the ROM, we placed a sensor of a magnetic 3D position measuring device (LIBERTY, Polhemus, USA), $15 \mathrm{~cm}$ above the cranial of the patella. To accurately perform the statistical analysis, the participants repeated the ASLR test three times and the mean value was calculated.

\section{Statistical analysis}

To compare the measurement value of muscular stiffness and ROM using the ASLR test in each intervention (before, immediately after, 15 minutes later, and 30 minutes later), one-way ANOVA was used. For the subtests, Dunnett's test was used for multiple comparisons. The level of significance was set at a $\mathrm{p}$-value $<0.05(\mathrm{p}<0.05)$. The statistical software used was SPSS for Windows (version 24.0; IBM Corp., Armonk, New York, USA).

\section{Results}

Twenty-four healthy male subjects (age, $33.0 \pm 8.4$ years; height, $174.4 \pm 7.5 \mathrm{~cm}$; weight, $66.6 \pm 7.9 \mathrm{~kg}$; $\mathrm{BMI}, 21.8 \pm 1.8)$ participated in this study. 
The results for muscular stiffness and ASLR tests are shown in Table 1. The muscular stiffness of the superficial and deep multifidus muscles was significantly lower at T1 than that before the intervention (T0). In terms of ROM, the ROM was significantly greater at T1 than at T0. Both measurement components showed significant differences between T0, T2, and T3.

Table 1

Sequential changes in muscle stiffness and ASLR

\begin{tabular}{|c|c|c|c|c|}
\hline & T0 & T1 & T2 & T3 \\
\hline \multicolumn{5}{|l|}{ Stiffness } \\
\hline Superficial multifidus & $5.42 \pm 9.87$ & $0.71 \pm 0.44^{\star}$ & $0.71 \pm 0.47^{*}$ & $0.67 \pm 0.38^{*}$ \\
\hline Deep multifidus & $12.9 \pm 18.1$ & $1.38 \pm 1.83^{*}$ & $0.99 \pm 0.60^{*}$ & $1.09 \pm 0.75^{\star}$ \\
\hline $\operatorname{ASLR}\left({ }^{\circ}\right)$ & $64.1 \pm 14.7$ & $69.7 \pm 16.5^{\star}$ & $68.9 \pm 18.5^{\star}$ & $65.9 \pm 16.7 *$ \\
\hline \multicolumn{5}{|c|}{ Values are represented as mean \pm standard deviation } \\
\hline \multicolumn{5}{|c|}{ *Significant difference from T0 value $(p<0.05)$. } \\
\hline \multicolumn{5}{|c|}{ ASLR, active straight leg raise } \\
\hline
\end{tabular}

\section{Discussion}

This study examined the immediate effects and the duration of multifidus muscular stiffness and ROM using the ASLR test after CRET therapy to the lower back. The muscular stiffness of the superficial and deep multifidus muscles after the intervention was significantly lesser and the ROM increased significantly compared to those before the intervention. The effects lasted for 30 minutes. These results showed that CRET not only causes pain reduction but also improves the stiffness and flexibility of the muscles. Muscular stiffness and decreased flexibility are causes of impairment [29,30]. Therefore, CRET may contribute to the prevention of muscle strain or low back pain and improve sports performance.

CRET reportedly improves pain and blood circulation after an injury. Thermal stimulation reduces pain directly or indirectly by inhibiting ischemia and muscle spasms and facilitating tissue healing [31, 32]. Stimulation of the thermal receptors cause vasodilation, and ischemic pain is reduced. This mechanism is considered to reduce the vasospasm. Vasodilation by thermotherapy facilitates tissue healing and an increase in the local pain threshold. CRET is an effective treatment for injured participants and is used in clinical settings; however, in this study, we showed improvement in the muscular stiffness and the ROM in healthy participants. It seems that the flexibility of the muscle and collagen tissue of the fascia was improved. The collagen soft tissue can undergo elastic deformation by thermal energy, and it increases and maintains its extensibility [33,34]. Knight et al. reported that deep heating by ultrasound on the plantaris muscles significantly increased dorsiflexion [35]. CRET also increased the ROM in the present study. Because ultrasound has a smaller probe and there is a risk of inflammation in the periosteum [36], CRET may be a safer option than ultrasound. The multifidus muscle and thoracolumbar fascia have 
muscle connections with the hamstrings via the sacral ligament or ischial tuberosity [37]. Therefore, the increased flexibility of the multifidus muscle and thoracolumbar fascia due to CRET improved the ROM. Improving the ROM is a vital aim in addition to raising the muscle temperature during warm-ups for sports events [21]. In particular, increased ROM can prevent injury and improve the splinting and kicking performance [38, 39].

With respect to decreased muscular stiffness, the stagnant body fluid might be removed by improving blood circulation. Muscular stiffness occurs through increased intramuscular pressure caused by stagnant body fluid [25]. In addition, muscular stiffness causes reduced ROM; therefore, CRET may contribute to increasing the ROM. The second factor for decreased muscular stiffness was an increase in the extensibility of the soft tissue including connective tissue with collagen fiber [40, 41]. As the temperature increases, the extensibility of collagen fibers increases; leading to a decrease in the viscoelasticity of muscle fibers and viscosity of the connective tissue [42]. Muscle flexibility was gained due to a greater extensibility of soft tissue.

The effect on muscular stiffness and ASLR remained for 30 minutes. Warm-ups before sports events is widely endorsed by athletes and coaches, and it is necessary to ensure optimal performance. However, excess active warm-ups may cause muscle fatigue and result in poor performance. Neiva et al. reported that warm-up was recommended for 15-25 minutes to prevent fatigue [43]. Utilization of CRET differs from active warm-up; it does not deplete energy and enables improvement of superficial and deep muscular stiffness and the ROM. Therefore, in this study, CRET could prevent injury and contribute to improved sports performance.

This study had several limitations. We included healthy young men; however, it is necessary to confirm the effect on other groups with different compositions such as varying age groups, athletes of various sports, or females. Second, it is unknown whether the same effect of CRET would be found in the other areas. Navarro-Ledesma et al. described that CRET intervention could change the elasticity of the supraspinatus tendon in healthy badminton athletes [44]. The insertion site of the supraspinatus tendon has insufficient blood supply and tissue degeneration or rupture may likely [45]; therefore, CRET therapy may be effective. On the contrary, the Achilles tendon or plantaris muscle needs to maintain stiffness to generate the running economy and speed $[46,47]$; therefore, improving flexibility may cause poor performance.

\section{Conclusion}

In this study, CRET intervention improved the multifidus muscle stiffness and ROM using the ASLR test, and the effect lasted for 30 minutes. The aim of the warm-up is to improve muscular stiffness, increase the ROM, raise the muscle temperature, and prevent injuries. CRET may be a useful therapy for passive warm-up in sports and clinical settings. Furthermore, CRET's effects on the actual sports performance, such as running or jumping, need to be studied. Active warm-up is also required after passive warm-up. 
Therefore, future studies should compare actual sports movement or combined usage with other warmup exercises.

\section{Abbreviations}

CRET

Capacitive and resistive electric transfer

ASLR

Active straight leg raise

ANOVA

Analysis of variance

BMI

Body mass index

T0

baseline

T1

Immediately after intervention

T2

After 15 minutes

T3

After 30 minutes

ROM

Range of motion

CET

Capacitive electrode transfer

RET

Resistive electrode transfer

\section{Declarations}

\section{Ethics approval and consent to participate}

This study was approved by the ethics committee of the Kanazawa Orthopedic Sports Medicine Clinic (kanazawa-OSMC-2021-004). Each participant signed an informed consent form, based on the requirements of the Helsinki Declaration.

Consent for publication

Not applicable

Availability of data and materials 
The datasets of the current study are available from the corresponding author on reasonable request.

\section{Competing interests}

The authors declare that they have no competing interest.

\section{Funding}

This research received no external funding.

\section{Authors' contributions}

TJ, TF and MW conceived and designed the experiments; TJ, YO and MW performed the experiments; TJ, YO and MW analyzed the data; JT and AS contributed reagents, materials, and analysis tools; TJ, AS and MW wrote the paper; MI and TF wrote, reviewed and edited the paper. All authors have read and agreed to the published version of the manuscript.

\section{Acknowledgments}

We are grateful to all individuals who gave their time and effort to participate in this study.

\section{References}

1. Gam AN, Johannsen F. Ultrasound therapy in musculoskeletal disorders: a meta-analysis. Pain. 1995;63:85-91.

2. van der Windt D, van der Heijden G, van den Berg SGM, Ter Riet G, de Winter AF, Bouter LM. Ultrasound therapy for musculoskeletal disorders: a systematic review. Pain. 1999;81:257-71.

3. Furlan RM, Giovanardi RS, Britto AT, Oliveira e Britto DB. The use of superficial heat for treatment of temporomandibular disorders: an integrative review. Codas. 2015;27:207-12.

4. Malanga GA, Yan N, Stark J. Mechanisms and efficacy of heat and cold therapies for musculoskeletal injury. Postgrad Med. 2015;127:57-65.

5. Nadler SF, Weingand K, Kruse RJ. The physiologic basis and clinical applications of cryotherapy and thermotherapy for the pain practitioner. Pain Physician. 2004;7:395-9.

6. Hayes BT, Merrick MA, Sandrey MA, Cordova ML. Three-MHz Ultrasound Heats Deeper Into the Tissues Than Originally Theorized. J AthI Train. 2004;39:230-4.

7. Tashiro Y, Hasegawa S, Yokota Y, Nishiguchi S, Fukutani N, Shirooka H, et al. Effect of Capacitive and Resistive electric transfer on haemoglobin saturation and tissue temperature. Int $\mathrm{J}$ Hyperthermia. 2017;33:696-702.

8. Yoshitake Y, Ue H, Miyazaki M, Moritani T. Assessment of lower-back muscle fatigue using electromyography, mechanomyography, and near-infrared spectroscopy. Eur J Appl Physiol. 2001;84:174-9. 
9. Baker RJ, Bell GW. The effect of therapeutic modalities on blood flow in the human calf. J Orthop Sports Phys Ther. 1991;13:23-7.

10. Robinson SE, Buono MJ. Effect of continuous-wave ultrasound on blood flow in skeletal muscle. Phys Ther. 1995;75:145-9. discussion 9-50.

11. Baker KG, Robertson VJ, Duck FA. A review of therapeutic ultrasound: biophysical effects. Phys Ther. 2001;81:1351-8.

12. Crockford GW, Hellon RF, Parkhouse J. Thermal vasomotor responses in human skin mediated by local mechanisms. J Physiol. 1962;161:10-20.

13. Wessman HC, Kottke FJ. The effect of indirect heating on peripheral blood flow, pulse rate, blood pressure, and temperature. Arch Phys Med Rehabil. 1967;48:567-76.

14. Sekins KM, Lehmann JF, Esselman P, Dundore D, Emery AF, deLateur BJ, et al. Local muscle blood flow and temperature responses to $915 \mathrm{MHz}$ diathermy as simultaneously measured and numerically predicted. Arch Phys Med Rehabil. 1984;65:1-7.

15. Batavia M. Contraindications for superficial heat and therapeutic ultrasound: do sources agree? Arch Phys Med Rehabil. 2004;85:1006-12.

16. Hui CF, Chan CW, Yeung HY, Lee KM, Qin L, Li G, et al. Low-intensity pulsed ultrasound enhances posterior spinal fusion implanted with mesenchymal stem cells-calcium phosphate composite without bone grafting. Spine (Phila Pa 1976). 2011;36:1010-6.

17. Kato S, Saitoh Y, Miwa N. Repressive effects of a capacitive-resistive electric transfer (CRet) hyperthermic apparatus combined with provitamin $\mathrm{C}$ on intracellular lipid-droplets formation in adipocytes. Int J Hyperthermia. 2013;29:30-7.

18. Takahashi K, Suyama T, Onodera M, Hirabayashi S, Tsuzuki N, Zhong-Shi L. Clinical Effects of Capacitive Electric Transfer Hyperthermia Therapy for Lumbago. Journal of Physical Therapy Science. 1999;11:45-51.

19. Kim GW, Won YH, Park SH, Seo JH, Kim DH, Lee HN, et al. Effects of a Newly Developed Therapeutic Deep Heating Device Using High Frequency in Patients with Shoulder Pain and Disability: A Pilot Study. Pain Res Manag. 2019;2019:8215371.

20. Tashiro Y, Suzuki Y, Nakayama Y, Sonoda T, Yokota Y, Kawagoe M, et al. The effect of Capacitive and Resistive electric transfer on non-specific chronic low back pain. Electromagn Biol Med. 2020;39:437-44.

21. McGowan CJ, Pyne DB, Thompson KG, Rattray B. Warm-Up Strategies for Sport and Exercise: Mechanisms and Applications. Sports Med. 2015;45:1523-46.

22. Grant ME, Steffen K, Palmer D. The usage of multidisciplinary physical therapies at the Rio de Janeiro 2016 Olympic Summer Games: an observational study. Braz J Phys Ther. 2021;25:262-70.

23. Grant ME, Steffen K, Glasgow P, Phillips N, Booth L, Galligan M. The role of sports physiotherapy at the London 2012 Olympic Games. Br J Sports Med. 2014;48:63-70. 
24. Murillo C, Falla D, Rushton A, Sanderson A, Heneghan NR. Shear wave elastography investigation of multifidus stiffness in individuals with low back pain. J Electromyogr Kinesiol. 2019;47:19-24.

25. Yanagisawa O, Niitsu M, Kurihara T, Fukubayashi T. Evaluation of human muscle hardness after dynamic exercise with ultrasound real-time tissue elastography: a feasibility study. Clin Radiol. 2011;66:815-9.

26. Yuri T, Mura N, Yuki I, Fujii H, Kiyoshige Y. Contractile property measurement of the torn supraspinatus muscle using real-time tissue elastography. J Shoulder Elbow Surg. 2018;27:1700-4.

27. Herrington L, Bendix K, Cornwell C, Fielden N, Hankey K. What is the normal response to structural differentiation within the slump and straight leg raise tests? Man Ther. 2008;13:289-94.

28. Hu H, Meijer OG, Hodges PW, Bruijn SM, Strijers RL, Nanayakkara PW, et al. Understanding the Active Straight Leg Raise (ASLR): an electromyographic study in healthy subjects. Man Ther. 2012;17:5317.

29. Roussel NA, Nijs J, Truijen S, Smeuninx L, Stassijns G. Low back pain: clinimetric properties of the Trendelenburg test, active straight leg raise test, and breathing pattern during active straight leg raising. J Manipulative Physiol Ther. 2007;30:270-8.

30. Hrysomallis C. Injury incidence, risk factors and prevention in Australian rules football. Sports Med. 2013;43:339-54.

31. Lehmann JF, Brunner GD, Stow RW. Pain threshold measurements after therapeutic application of ultrasound, microwaves and infrared. Arch Phys Med Rehabil. 1958;39:560-5.

32. Benson TB, Copp EP. The effects of therapeutic forms of heat and ice on the pain threshold of the normal shoulder. Rheumatol Rehabil. 1974;13:101-4.

33. Gersten JW. Effect of ultrasound on tendon extensibility. Am J Phys Med. 1955;34:362-9.

34. Lehmann JF, Masock AJ, Warren CG, Koblanski JN. Effect of therapeutic temperatures on tendon extensibility. Arch Phys Med Rehabil. 1970;51:481-7.

35. Knight CA, Rutledge CR, Cox ME, Acosta M, Hall SJ. Effect of superficial heat, deep heat, and active exercise warm-up on the extensibility of the plantar flexors. Phys Ther. 2001;81:1206-14.

36. Cameron MH. Physical agents in rehabilitation: from research to practice. St. Louis: Saunders Elsevier; 2009.

37. Myers TW. Anatomy trains. Edinburgh: Churchill Livingstone/Elsevier; 2009.

38. Amiri-Khorasani M, Abu Osman NA, Yusof A. Acute effect of static and dynamic stretching on hip dynamic range of motion during instep kicking in professional soccer players. J Strength Cond Res. 2011;25:1647-52.

39. García-Pinillos F, Ruiz-Ariza A, Moreno del Castillo R, Latorre-Román P. Impact of limited hamstring flexibility on vertical jump, kicking speed, sprint, and agility in young football players. J Sports Sci. 2015;33:1293-7.

40. Lehmann JF, Warren CG, Scham SM. Therapeutic heat and cold. Clin Orthop Relat Res. 1974:20745. 
41. Strickler T, Malone T, Garrett WE. The effects of passive warming on muscle injury. Am J Sports Med. 1990;18:141-5.

42. Mutungi G, Ranatunga KW. Temperature-dependent changes in the viscoelasticity of intact resting mammalian (rat) fast- and slow-twitch muscle fibres. J Physiol. 1998;508(Pt 1):253-65.

43. Neiva HP, Marques MC, Barbosa TM, Izquierdo M, Marinho DA. Warm-up and performance in competitive swimming. Sports Med. 2014;44:319-30.

44. Navarro-Ledesma S, Gonzalez-Muñoz A. Short-term effects of 448 kilohertz radiofrequency stimulation on supraspinatus tendon elasticity measured by quantitative ultrasound elastography in professional badminton players: a double-blinded randomized clinical trial. Int J Hyperthermia. 2021;38:421-7.

45. Fenwick SA, Hazleman BL, Riley GP. The vasculature and its role in the damaged and healing tendon. Arthritis Res. 2002;4:252-60.

46. Fletcher JR, Esau SP, Maclntosh BR. Changes in tendon stiffness and running economy in highly trained distance runners. Eur J Appl Physiol. 2010;110:1037-46.

47. Lai A, Schache AG, Lin YC, Pandy MG. Tendon elastic strain energy in the human ankle plantarflexors and its role with increased running speed. J Exp Biol. 2014;217:3159-68.

\section{Figures}

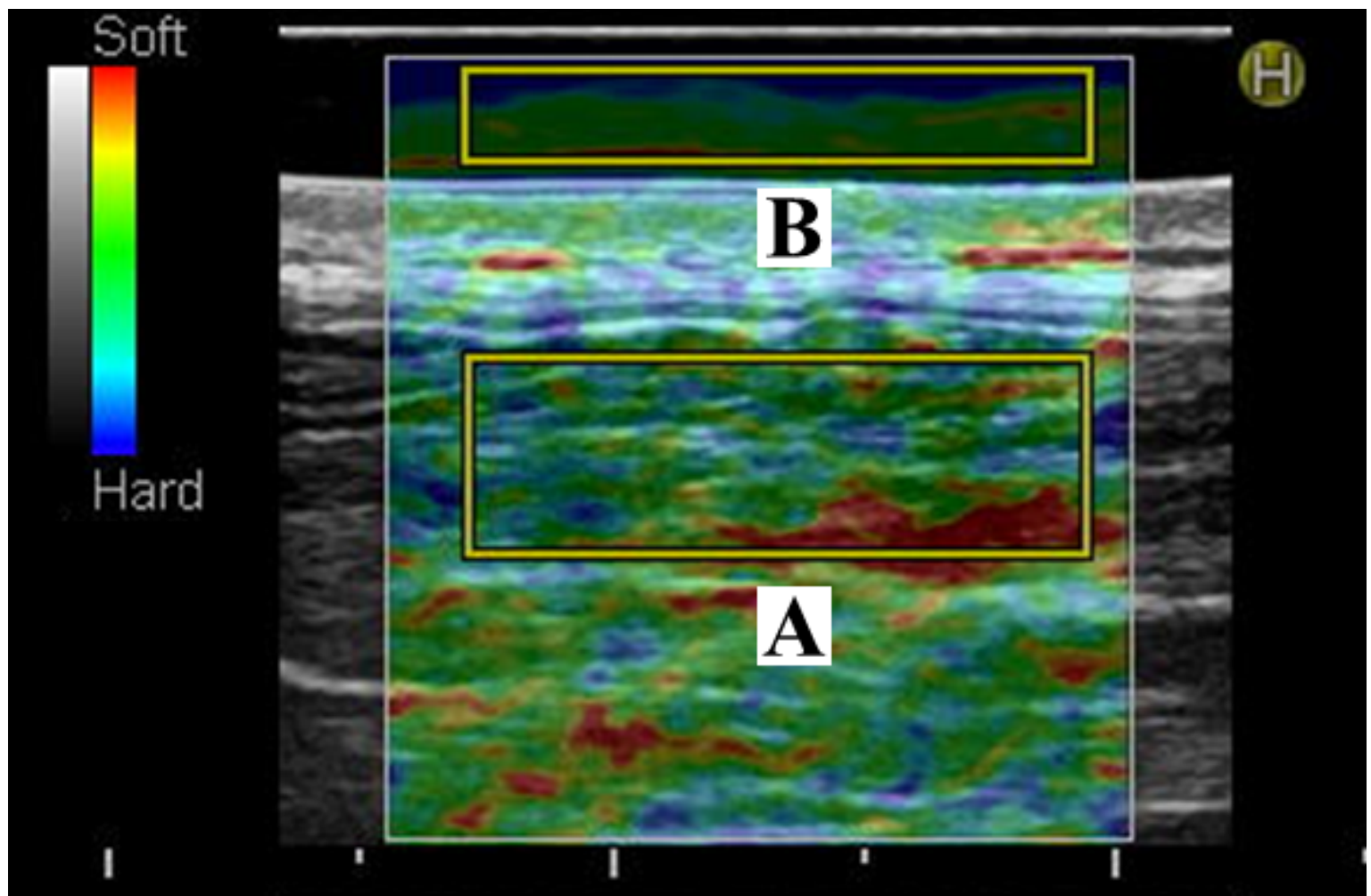

Figure 1

Representative elastography images of the superficial multifidus muscles(A) and the acoustic coupler(B) 